\title{
Isospin and Local Space-Time Rotations
}

\author{
J. G. VALATIN \\ Department of Physics, Queen Mary College, University of London
}

Received June 11, 1968

\begin{abstract}
An 8-component spinor field carries with itself a large number of 4-vector currents and invariants, the relationships between which are analysed. The vector densities can be grouped into a number of orthogonal frames, which describe tetrad fields. Two of the tetrads, related to each other by charge conjugation, connect isospin transformations directly with local space-time rotations. The main tetrad planes determine geometrical configurations of considerable symmetry. The bilinear invariants define angles and hyperbolic angles which appear directly in the rotations and Lorentz transformations connecting the tetrads.
\end{abstract}

\section{Introduction}

Recent interest in the algebra of currents has again drawn attention to the possibility that the 4-vector densities which can be formed from spinorial fields may play a basic role in the description of elementary particles. These 4-vector densities are known to satisfy a number of relationships which, mainly for $c$-number spinors of 4-components, have been the subject of many investigations $[1-17]$.

The interest of this paper will be in the relationships between 4-vectors and invariants carried by an 8-component spinor field which may describe isospin. An extension of the results to a larger number of components will be seen to be straightforward. Only $c$-number fields will be considered, but the way in which the relationships are obtained will indicate the existence of related relationships for operator fields.

These $c$-number fields may enter into a physical theory in many other ways than the description of states of non-interacting particles. Any matrix element of a spinor field operator of the Heisenberg representation is a $c$-number spinor field, and has all the characteristics investigated in this paper. Such $c$-number fields or derived quantities can play the part of sources or variables, functionals of which describe a quantised theory. A classical or semi-classical theory of $c$-number fields has concepts which are easier to visualise, and can be studied for its own sake. One may try to find first a suitable new form of classical theory before attempting quantisation. There may be other directions in which new concepts appearing for $c$-number quantities may suggest ways of progress.

13 Commun. math. Phys., Vol.10 
For a 4-component Dirac wave function the relationships between the bilinear covariants have been noted and investigated from the early days of quantum mechanics, by Darwin [1], Fook [2], PaULI [3], LouIS DE BRogLIE and his school [4-9], and many others [10-14]. With an increase of the number of degrees of freedom, the number of bilinear densities increases considerably and the field has been explored relatively much less [15-17]. The relationships studied in the case of a Dirac wave function are of two different types. There are those which are of purely algebraic character, and those which always contain derivatives and are obtained with the help of the Dirac equation. Examples of the second type are the continuity equation of the electric current, the decomposition of the vector current into a contribution of the Klein-Gordon type and a divergence of the electromagnetic polarisation tensor, or an analogous axial current relationship due to Proca [5]. These relationships are very suggestive and some of them may be of much more general validity than indicated by the special dynamical origin of their derivation. The interest of the present paper will be, however, exclusively in the purely algebraic and geometrical relationships which are valid for any spinor fields irrespective of any dynamics.

The 16 real bilinear densities formed from a Dirac wave function represent only 7 independent real data. An arbitrary phase factor of the Dirac wave function can still be added at each point to obtain the 8 real data needed to replace the 4 complex components of the wave function. If one restricts attention to quantities bilinear in $\bar{\psi}$ and $\psi$, one has to include one of the densities defined with derivatives in order to give a complete set of data. The alternative is to consider simultaneously densities quadratic in $\psi$, or quadratic in $\bar{\psi}$, or equivalently densities bilinear in $\bar{\psi}_{c}, \psi$ or $\bar{\psi}, \psi_{c}$. Instead of two 4-vectors, one finds in this way four real 4-vectors which form an orthogonal frame and define a tetrad field. This has been noticed and discussed by WhItTAKER [11], RUSE [12], Aymard [9], Takabayasi [14]. The inclusion of all the four vectors of the tetrad is basic from the algebraic or geometrical point of view, and indicates a possible role for 'anomalous' densities or expectation values, similar to that in the theory of superconductivity.

The structure and relationships of the vectors is especially simple $[9,11,12]$ in terms of the 2-component spinors of the Weyl representation. This will be consistently exploited and emphasised throughout the paper. The symmetric role played by the 2-component spinors defining $\psi$ and $\bar{\psi}$ may also be indicative when formulating a theory of spinor fields.

The next section gives a short review of some of the relationships between quantities connected with a 4-component Dirac spinor. Notation and relationships are set up in a form in which they can be immediately 
applied and generalised to the larger number of quantities defined in the presence of isospin, which are introduced in the following section. The definition of 4-vectors and invariants of the more general case is spelt out in some detail, so that their meaning and spinorial construction can be easily referred to. The many similar relationships connecting these quantities need not then be written out separately, as they can be obtained from each other by an interchange of symbols which can be simply indicated.

The basic null-vectors that can be formed from four 2-component spinors determine in the general case six tetrads. An 8-component spinor field describes in this way in general six tetrad fields. The six complex invariants can be decomposed into amplitudes and phase factors. The amplitudes describe positive densities and give the lengths of the current vectors. The phase factors define angular variables which describe multivalued functions determined only up to integer multiples of $2 \pi$. The line integrals of the gradients of the angle variables would give integer numbers which are in correlation with the zeros of the amplitudes. In analysing the relative orientation of the tetrads, differences of these phase angles will appear as actual geometrical angles, whereas the amplitudes define hyperbolic angles of Lorentz transformations.

\section{4-Component Spinors}

A 4-component Dirac spinor can be written in the Weyl representation $[9,18]$ in the form

$$
\psi=(\xi, \tilde{\eta})
$$

where the 2-component spinors

and

$$
\xi:\left(\xi_{1}, \xi_{2}\right), \quad \eta:\left(\eta_{1}, \eta_{2}\right)
$$

$$
\tilde{\eta}:\left(\tilde{\eta}_{1}, \tilde{\eta}_{2}\right) \quad \text { with } \quad \tilde{\eta}_{1}=-\eta_{2}^{*}, \quad \tilde{\eta}_{2}=\eta_{1}^{*}
$$

transform in covariant and contravariant ways.

To have a definite representation in mind, $2 \times 2$ Pauli matrices

$$
\sigma_{0}:\left(\begin{array}{ll}
1 & 0 \\
0 & 1
\end{array}\right), \quad \sigma_{1}:\left(\begin{array}{ll}
0 & 1 \\
1 & 0
\end{array}\right), \quad \sigma_{2}:\left(\begin{array}{rr}
0 & -i \\
i & 0
\end{array}\right), \quad \sigma_{3}:\left(\begin{array}{rr}
1 & 0 \\
0 & -1
\end{array}\right)
$$

can be chosen acting on the components of $\xi$ or $\tilde{\eta}$. Analogous matrices

$$
\varrho_{0}:\left(\begin{array}{ll}
1 & 0 \\
0 & 1
\end{array}\right), \quad \varrho_{1}:\left(\begin{array}{ll}
0 & 1 \\
1 & 0
\end{array}\right), \quad \varrho_{2}:\left(\begin{array}{rr}
0 & -i \\
i & 0
\end{array}\right), \quad \varrho_{3}:\left(\begin{array}{lr}
1 & 0 \\
0 & -1
\end{array}\right)
$$

can be introduced to act on the two spinor components of $\psi$. With the choice

$$
\gamma_{0}=\sigma_{0} \varrho_{1}, \quad \gamma_{j}=\frac{1}{i} \sigma_{j} \varrho_{2}, \quad j=1,2,3, \quad \gamma_{5}=\sigma_{0} \varrho_{3}
$$


the matrices $\gamma_{0}, \gamma_{5}$ are Hermitian, $\gamma_{1}, \gamma_{2}, \gamma_{3}$ are anti-Hermitian and they all anti-commute.

With

$$
\psi^{*}=\left(\xi^{*}, \tilde{\eta}^{*}\right), \quad \bar{\psi}=\psi^{*} \gamma_{0}=\left(\tilde{\eta}^{*}, \xi^{*}\right),
$$

the charge conjugation matrix $C$ which defines $\psi_{c}=C \psi^{*}$ can be chosen to be $C=\left(i \sigma_{2}\right)\left(i \varrho_{2}\right)=-i \gamma_{2}$, so that

$$
\psi_{c}=(\eta, \tilde{\xi}), \quad \bar{\psi}_{c}=\left(\tilde{\xi}^{*}, \eta^{*}\right) .
$$

A charge conjugation $\psi \rightleftarrows \psi_{c}$ corresponds in this way to an interchange of $\xi$ and $\eta$.

The Dirac spinor $\psi$ defines the basic null-vectors

$$
\begin{array}{ll}
R_{\mu}=\bar{\psi} \gamma_{\mu} \frac{1}{2}\left(1+\gamma_{5}\right) \psi, & L_{\mu}=\bar{\psi} \gamma_{\mu} \frac{1}{2}\left(1-\gamma_{5}\right) \psi, \\
Z_{\mu}=\bar{\psi} \gamma_{\mu} \frac{1}{2}\left(1+\gamma_{5}\right) \psi_{c}, & Z_{\mu}^{*}=\bar{\psi}_{c} \gamma_{\mu} \frac{1}{2}\left(1+\gamma_{5}\right) \psi .
\end{array}
$$

With

$$
\frac{1}{2}\left(1+\gamma_{5}\right) \psi=(\xi, 0), \quad \frac{1}{2}\left(1-\gamma_{5}\right) \psi=(0, \tilde{\eta}),
$$

these can be expressed in terms of 2-component spinors in the form

$$
\begin{array}{ll}
R_{\mu}=\xi^{*} \sigma_{\mu} \xi, & L_{\mu}=\eta^{*} \sigma_{\mu} \eta \\
Z_{\mu}=\xi^{*} \sigma_{\mu} \eta, & Z_{\mu}^{*}=\eta^{*} \sigma_{\mu} \xi,
\end{array}
$$

where $\tilde{\eta}, \tilde{\xi}$ have been eliminated with the help of the relations $\tilde{\eta}^{*} \tilde{\eta}$ $=\eta^{*} \eta,-\tilde{\eta}^{*} \sigma_{j} \tilde{\eta}=\eta^{*} \sigma_{j} \eta, j=1,2,3$. Since the matrices $\sigma_{\mu}$ are Hermitian, the form (5c) of $Z_{\mu}, Z_{\mu}^{*}$ shows that the two vectors are complex conjugate. One can introduce complex invariants

which give

$$
\frac{1}{2} \Omega=\bar{\psi} \frac{1}{2}\left(1+\gamma_{5}\right) \psi, \frac{1}{2} \Omega^{*}=\bar{\psi} \frac{1}{2}\left(1-\gamma_{5}\right) \psi,
$$

$$
\bar{\psi} \psi=\frac{1}{2}\left(\Omega+\Omega^{*}\right), \quad \bar{\psi} \gamma_{5} \psi=\frac{1}{2 i}\left(\Omega-\Omega^{*}\right),
$$

and for which one finds

$$
\begin{aligned}
\frac{1}{2} \Omega & =\left|\begin{array}{ll}
\eta_{1} & \eta_{2} \\
\xi_{1} & \xi_{2}
\end{array}\right|=\eta \times \xi, \\
\frac{1}{2} \Omega^{*} & =\left|\begin{array}{ll}
\eta_{1}^{*} & \eta_{2}^{*} \\
\xi_{1}^{*} & \xi_{2}^{*}
\end{array}\right|=\eta^{*} \times \xi^{*} .
\end{aligned}
$$

The length and scalar products of vectors formed from 2-component spinors can be expressed in terms of invariants of this form. If $\alpha, \beta, \alpha^{\prime}, \beta^{\prime}$ are four 2-component spinors, and one forms the vectors

$$
U_{\mu}=\alpha^{*} \sigma_{\mu} \beta, \quad U_{\mu}^{\prime}=\alpha^{*} \sigma_{\mu} \beta^{\prime},
$$


with the Pauli matrices (2a) one has

$$
\begin{array}{ll}
\frac{1}{2}\left(U_{0}+U_{3}\right)=\alpha_{1}^{*} \beta_{1}, & \frac{1}{2}\left(U_{0}-U_{3}\right)=\alpha_{2}^{*} \beta_{2}, \\
\frac{1}{2}\left(U_{1}+i U_{2}\right)=\alpha_{1}^{*} \beta_{2}, & \frac{1}{2}\left(U_{1}-i U_{2}\right)=\alpha_{2}^{*} \beta_{1},
\end{array}
$$

and similar expressions related to $U_{\mu}^{\prime}$. If one writes the scalar product of the two vectors in the form

$$
\begin{aligned}
U^{\mu} U_{\mu}^{\prime}= & \frac{1}{2}\left\{\left(U_{0}+U_{3}\right)\left(U_{0}^{\prime}-U_{3}^{\prime}\right)+\left(U_{0}-U_{3}\right)\left(U_{0}^{\prime}-U_{3}^{\prime}\right)\right. \\
& \left.-\left(U_{1}+i U_{2}\right)\left(U_{1}^{\prime}-i U_{2}^{\prime}\right)-\left(U_{1}-i U_{2}\right)\left(U_{1}^{\prime}+i U_{2}^{\prime}\right)\right\}
\end{aligned}
$$

the four terms exhibit explicitly the identity

$$
\left(\alpha^{*} \sigma^{\mu} \beta\right)\left(\alpha^{*} \sigma_{\mu} \beta^{\prime}\right)=2\left|\begin{array}{cc}
\alpha_{1}^{*} & \alpha_{2}^{*} \\
\alpha_{2}^{\prime *} & \alpha_{1}^{\prime *}
\end{array}\right|\left|\begin{array}{cc}
\beta_{1} & \beta_{2} \\
\beta_{1}^{\prime} & \beta_{2}^{\prime}
\end{array}\right| .
$$

For the vectors $(5 \mathrm{~b}, \mathrm{c})$, the identity gives directly

$$
\begin{aligned}
R^{\mu} R_{\mu} & =0, \quad L^{\mu} L_{\mu}=0, \quad Z^{\mu} Z_{\mu}=0, \\
2 R^{\mu} L_{\mu} & =\Omega^{*} \Omega, \quad 2 Z^{\mu} Z_{\mu}^{*}=-\Omega^{*} \Omega, \\
R^{\mu} Z_{\mu} & =0, \quad L^{\mu} Z_{\mu}=0 .
\end{aligned}
$$

The two real null-vectors $R_{\mu}, L_{\mu}$ and the complex null-vector $Z_{\mu}$ define four real vectors

$$
\begin{aligned}
& V_{\mu}=R_{\mu}+L_{\mu}=\bar{\psi} \gamma_{\mu} \psi, \\
& W_{\mu}=R_{\mu}-L_{\mu}=\bar{\psi} \gamma_{\mu} \gamma_{5} \psi, \\
& X_{\mu}=Z_{\mu}+Z_{\mu}^{*}, \\
& Y_{\mu}=\frac{1}{i}\left(Z-Z_{\mu}^{*}\right) .
\end{aligned}
$$

(The expressions vector and invariant are used throughout in the sense of proper Lorentz transformations.) According to the relationships $(8 a, b, c)$, these vectors are orthogonal and the absolute value of their length is equal. With an abbreviated notation for the scalar product, $V^{\mu} V_{\mu}=V^{2}$, one has

$$
\begin{aligned}
V^{2} & =-W^{2}=-X^{2}=-Y^{2}=|\Omega|^{2}, \\
V \cdot W & =V \cdot X=V \cdot Y=W \cdot X=W \cdot Y=X \cdot Y=0 .
\end{aligned}
$$

For $|\Omega| \neq 0$, one can define unit vectors $v, w, x, y$ by the equations

$$
V_{\mu}=|\Omega| v_{\mu}, \quad W_{\mu}=|\Omega| w_{\mu}, \quad X_{\mu}=|\Omega| x_{\mu}, \quad Y_{\mu}=|\Omega| y_{\mu} .
$$

These form an orthonormal system, with

$$
\begin{gathered}
v^{2}=-w^{2}=-x^{2}=-y^{2}=1, \\
v \cdot w=v \cdot x=v \cdot y=w \cdot x=w: y=x \cdot y=0,
\end{gathered}
$$


and define a tetrad, (Vierbein). As a function of the space-time coordinates, one obtains a tetrad field. At a given point, the tetrads are determined by 6 independent real numbers. If one defines the angle $\theta$ by

$$
\Omega=|\Omega| \exp (i \theta)
$$

the two real data $|\Omega|$ and $\theta$ can be added to obtain eight independent real data which are equivalent to the four complex components of $\psi$. Alternatively, one could consider as basic quantities the four currents $V, W$, $X, Y$ and the invariant $\Omega$, together with the relationships $(10 \mathrm{a}, \mathrm{b})$. The 6 -vector densities formed with $\bar{\psi}, \psi$ can be expressed by means of the vectors and invariants. For completeness, the relevant identities are given in the Appendix.

\section{Tetrads and Isospin}

For an 8-component spinor, one can write

with

$$
\psi=\left(\psi_{p}, \psi_{n}\right)
$$

Defining $2 \times 2$ isospin matrices $\tau_{0}, \tau_{1}, \tau_{2}, \tau_{3}$ in the same way as the matrices (2a), $(2 \mathrm{~b})$, these act on the two components $\psi_{p}, \psi_{n}$ of $\psi$. For

$$
\tau_{p}=\frac{1}{2}\left(1+\tau_{3}\right), \quad \tau_{n}=\frac{1}{2}\left(1-\tau_{3}\right), \quad \tau_{ \pm}=\frac{1}{2}\left(\tau_{1} \pm i \tau_{2}\right)
$$

the chosen matrices are

$$
\tau_{p}:\left(\begin{array}{ll}
1 & 0 \\
0 & 0
\end{array}\right), \quad \tau_{n}:\left(\begin{array}{ll}
0 & 0 \\
0 & 1
\end{array}\right), \quad \tau_{+}:\left(\begin{array}{ll}
0 & 1 \\
0 & 0
\end{array}\right), \quad \tau_{-}:\left(\begin{array}{ll}
0 & 0 \\
1 & 0
\end{array}\right) .
$$

Corresponding to the definition $(4 \mathrm{a}, \mathrm{b})$ of $R, L, Z, Z^{*}$, one obtains four times as many basic null-vectors,

$$
\begin{array}{ll}
\left(R_{p}\right)_{\mu}=\bar{\psi} \gamma_{\mu} \frac{1}{2}\left(1+\gamma_{5}\right) \tau_{p} \psi, & \left(R_{n}\right)_{\mu}=\bar{\psi} \gamma_{\mu} \frac{1}{2}\left(1+\gamma_{5}\right) \tau_{n} \psi, \\
\left(R_{+}\right)_{\mu}=\bar{\psi} \gamma_{\mu} \frac{1}{2}\left(1+\gamma_{5}\right) \tau_{+} \psi, & \left(R_{-}\right)_{\mu}=\bar{\psi} \gamma_{\mu} \frac{1}{2}\left(1+\gamma_{5}\right) \tau_{-} \psi, \\
\left(L_{p}\right)_{\mu}=\bar{\psi} \gamma_{\mu} \frac{1}{2}\left(1-\gamma_{5}\right) \tau_{p} \psi, & \left(L_{n}\right)_{\mu}=\bar{\psi} \gamma_{\mu} \frac{1}{2}\left(1-\gamma_{5}\right) \tau_{n} \psi, \\
\left(L_{+}\right)_{\mu}=\bar{\psi} \gamma_{\mu} \frac{1}{2}\left(1-\gamma_{5}\right) \tau_{+} \psi, & \left(L_{-}\right)_{\mu}=\bar{\psi} \gamma_{\mu} \frac{1}{2}\left(1-\gamma_{5}\right) \tau_{-} \psi, \\
\left(Z_{p}\right)_{\mu}=\bar{\psi} \gamma_{\mu} \frac{1}{2}\left(1+\gamma_{5}\right) \tau_{p} \psi_{c}, & \left(Z_{p}^{*}\right)_{\mu}=\bar{\psi}_{c} \gamma_{\mu} \frac{1}{2}\left(1+\gamma_{5}\right) \tau_{p} \psi, \\
\left(Z_{n}\right)_{\mu}=\bar{\psi} \gamma_{\mu} \frac{1}{2}\left(1+\gamma_{5}\right) \tau_{n} \psi_{c}, & \left(Z_{n}^{*}\right)_{\mu}=\bar{\psi}_{c} \gamma_{\mu} \frac{1}{2}\left(1+\gamma_{5}\right) \tau_{n} \psi, \\
\left(Z_{+}\right)_{\mu}=\bar{\psi} \gamma_{\mu} \frac{1}{2}\left(1+\gamma_{5}\right) \tau_{-} \psi_{c}, & \left(Z_{+}^{*}\right)_{\mu}=\bar{\psi}_{c} \gamma_{\mu} \frac{1}{2}\left(1+\gamma_{5}\right) \tau_{+} \psi, \\
\left(Z_{-}\right)_{\mu}=\bar{\psi} \gamma_{\mu} \frac{1}{2}\left(1+\gamma_{5}\right) \tau_{+} \psi_{c}, & \left(Z_{-}^{*}\right)_{\mu}=\bar{\psi}_{c} \gamma_{\mu} \frac{1}{2}\left(1+\gamma_{5}\right) \tau_{-} \psi
\end{array}
$$


With these definitions, the vectors $Z$ and $Z^{*}$ are complex conjugate. Note that the role of $\tau_{+}$and $\tau_{-}$is interchanged in the definition of $Z_{+}$ and $Z_{-}$, but not in $Z_{+}^{*}, Z_{-}^{*}$.

Instead of the complex invariant $\Omega$ of a 4-component spinor, one finds six invariants,

$$
\begin{array}{ll}
\frac{1}{2} \Omega_{p}=\bar{\psi} \frac{1}{2}\left(1+\gamma_{5}\right) \tau_{p} \psi, & \frac{1}{2} \Omega_{n}=\bar{\psi} \frac{1}{2}\left(1+\gamma_{5}\right) \tau_{n} \psi \\
\frac{1}{2} \Omega_{+}=\bar{\psi} \frac{1}{2}\left(1+\gamma_{5}\right) \tau_{+} \psi, & \frac{1}{2} \Omega_{-}=\bar{\psi} \frac{1}{2}\left(1+\gamma_{5}\right) \tau_{-} \psi \\
\frac{1}{2} \Omega_{R}=\bar{\psi}_{c} \frac{1}{2}\left(1+\gamma_{5}\right) \tau_{+} \psi, & \frac{1}{2} \Omega_{L}=\bar{\psi} \frac{1}{2}\left(1+\gamma_{5}\right) \tau_{-} \psi_{c} .
\end{array}
$$

One could write out similar explicit definitions for the complex conjugate $\frac{1}{2} \Omega^{*}-s$. In the definition (14c) of $\Omega_{R}, \Omega_{L}$, one could replace $\tau_{ \pm}$by $\pm \frac{1}{2} i \tau_{2}$.

In terms of the 2-component spinors $\xi_{p}, \eta_{p}, \xi_{n}, \eta_{n}$ the null-vectors $(13 a-c)$ can be expressed as

$$
\begin{array}{ll}
\left(R_{p}\right)_{\mu}=\xi_{p}^{*} \sigma_{\mu} \xi_{p}, & \left(R_{n}\right)_{\mu}=\xi_{n}^{*} \sigma_{\mu} \xi_{n}, \\
\left(R_{+}\right)_{\mu}=\xi_{p}^{*} \sigma_{\mu} \xi_{n}, & \left(R_{-}\right)_{\mu}=\xi_{n}^{*} \sigma_{\mu} \xi_{p}, \\
\left(L_{p}\right)_{\mu}=\eta_{p}^{*} \sigma_{\mu} \eta_{p}, & \left(L_{n}\right)_{\mu}=\eta_{n}^{*} \sigma_{\mu} \eta_{n}, \\
\left(L_{-}\right)_{\mu}=\eta_{p}^{*} \sigma_{\mu} \eta_{n}, & \left(L_{+}\right)_{\mu}=\eta_{n}^{*} \sigma_{\mu} \eta_{p}, \\
\left(Z_{p}\right)_{\mu}=\xi_{p}^{*} \sigma_{\mu} \eta_{p}, & \left(Z_{p}^{*}\right)_{\mu}=\eta_{p}^{*} \sigma_{\mu} \xi_{p}, \\
\left(Z_{n}\right)_{\mu}=\xi_{n}^{*} \sigma_{\mu} \eta_{n}, & \left(Z_{n}^{*}\right)_{\mu}=\eta_{n}^{*} \sigma_{\mu} \xi_{n}, \\
\left(Z_{+}\right)_{\mu}=\xi_{n}^{*} \sigma_{\mu} \eta_{p}, & \left(Z_{+}^{*}\right)_{\mu}=\eta_{p}^{*} \sigma_{\mu} \xi_{n}, \\
\left(Z_{-}\right)_{\mu}=\xi_{p}^{*} \sigma_{\mu} \eta_{n}, & \left(Z_{-}^{*}\right)_{\mu}=\eta_{n}^{*} \sigma_{\mu} \xi_{p} .
\end{array}
$$

With the identity $(7 \mathrm{~d})$, one can immediately see that all these vectors are indeed null-vectors. Their scalar products can be obtained with the help of the invariants $(14 a, b, c)$ and their complex conjugates. With the notation $(6 \mathrm{c})$ of the determinants, the invariants $\frac{1}{2} \Omega$ can be expressed in the form

$$
\begin{array}{ll}
\frac{1}{2} \Omega_{p}=\eta_{p} \times \xi_{p}, & \frac{1}{2} \Omega_{n}=\eta_{n} \times \xi_{n}, \\
\frac{1}{2} \Omega_{+}=\eta_{p} \times \xi_{n}, & \frac{1}{2} \Omega_{-}=\eta_{n} \times \xi_{p}, \\
\frac{1}{2} \Omega_{R}=\xi_{p} \times \xi_{n}, & \frac{1}{2} \Omega_{L}=\eta_{n} \times \eta_{p} .
\end{array}
$$


There are $\left(\begin{array}{l}4 \\ 2\end{array}\right)=6$ ways to select a pair from the four 2-component spinors $\xi_{p}, \eta_{p}, \xi_{n}, \eta_{n}$, and this leads to these 6 invariants. If one combines 4 null-vectors from $(15 \mathrm{a}, \mathrm{b}, \mathrm{c})$ which are built up from the same pair of 2-component spinors, one obtains an orthogonal tetrad, and 6 such tetrads can be constructed. In terms of 2-component spinors, both the number of covariants, and their relationships reveal a strong combinatorial background. The real null vectors $R_{p}, R_{n}, L_{p}, L_{n}$ which depend only on the components of a single spinor and their complex conjugate, will each take part in the building up of three tetrads, the remaining $2 \times 6=12$ complex null vectors enter pairwise into one of the tetrads only. One obtains in this way the 6 tetrads

$$
\begin{array}{ll}
V_{p}=R_{p}+L_{p}, \quad W_{p}=R_{p}-L_{p}, \\
X_{p}=Z_{p}+Z_{p}^{*}, \quad Y_{p}=\frac{1}{i}\left(Z_{p}-Z_{p}^{*}\right), \\
V_{n}=R_{n}+L_{n}, \quad W_{n}=R_{n}-L_{n}, \\
X_{n}=Z_{n}+Z_{n}^{*}, \quad Y_{n}=\frac{1}{i}\left(Z_{n}-Z_{n}^{*}\right), \\
V_{+}=R_{n}+L_{p}, \quad W_{+}=R_{n}-L_{p}, \\
X_{+}=Z_{+}+Z_{+}^{*}, \quad Y_{+}=\frac{1}{i}\left(Z_{+}-Z_{+}^{*}\right), \\
V_{-}=R_{p}+L_{n}, \quad W_{-}=R_{p}-L_{n}, \\
X_{-}=Z_{-}+Z_{-}^{*}, \quad Y_{-}=\frac{1}{i}\left(Z_{-}-Z_{-}^{*}\right), \\
V_{R}=R_{p}+R_{n}, & W_{R}=R_{p}-R_{n}, \\
X_{R}=R_{+}+R_{-}, & Y_{R}=\frac{1}{i}\left(R_{+}-R_{-}\right), \\
V_{L}=L_{p}+L_{n}, & W_{L}=L_{p}-L_{n}, \\
X_{L}=L_{-}+L_{+}, & Y_{L}=\frac{1}{i}\left(L_{-}-L_{+}\right) .
\end{array}
$$

The four current vectors entering into each tetrad are in the same relationship to each other as the vectors $(9 \mathrm{a}-\mathrm{d})$, since they are constructed from two 2-component spinors in exactly the same way. The length of the vectors within each tetrad is determined by the invariant $\Omega$ with the same suffix, and the relationships $(10 \mathrm{a}, \mathrm{b})$ remain valid with each of the six suffixes. If the invariants are non-vanishing, one can introduce unit vectors according to the equations (11a), and with the 
definition (11d) of the phase angles, one obtains the unit vectors and scalars

$$
\begin{array}{ll}
v_{p}, w_{p}, x_{p}, y_{p}, & \left|\Omega_{p}\right|, \theta_{p}, \\
v_{n}, w_{n}, x_{n}, y_{n}, & \left|\Omega_{n}\right|, \theta_{n}, \\
v_{+}, w_{+}, x_{+}, y_{+}, & \left|\Omega_{+}\right|, \theta_{+}, \\
v_{-}, w_{-}, x_{-}, y_{-}, & \left|\Omega_{-}\right|, \theta_{-}, \\
v_{R}, w_{R}, x_{R}, y_{R}, & \left|\Omega_{R}\right|, \theta_{R}, \\
v_{L}, w_{L}, x_{L}, y_{L}, & \left|\Omega_{L}\right|, \theta_{L} .
\end{array}
$$

There are a great number of relationships connecting these quantities. One possible way to select 16 independent real data would be to give two tetrads of unit vectors with the related invariants, for instance the data $(18 \mathrm{e}, \mathrm{f})$, or to give the 6 data of one tetrad of unit vectors together with 10 independent real data determined by the complex invariants. These would uniquely determine the 8-component spinor $\psi$.

According to the expressions $(16 a-c)$ of the invariants, the nonvanishing of all 6 invariants $\Omega$ means that the four 2 -component spinors are pairwise linearly independent. This is the general case, and there are some important special cases. If the number of pairwise linearly independent spinors is three, there are only three different tetrads. If this number is two there is only one tetrad, and a single 2-component spinor defines only a null-vector.

The connection between isospin transformations and the tetrads $(17 \mathrm{e}, \mathrm{f})$ is especially remarkable. From the definitions, one has

$$
\begin{aligned}
\left(V_{R}\right)_{\mu} & =\bar{\psi} \gamma_{\mu} \frac{1}{2}\left(1+\gamma_{5}\right) \tau_{0} \psi, & \left(V_{L}\right)_{\mu} & =\bar{\psi} \gamma_{\mu} \frac{1}{2}\left(1-\gamma_{5}\right) \tau_{0} \psi, \\
\left(X_{R}\right)_{\mu} & =\bar{\psi} \gamma_{\mu} \frac{1}{2}\left(1+\gamma_{5}\right) \tau_{1} \psi, & \left(X_{L}\right)_{\mu} & =\bar{\psi} \gamma_{\mu} \frac{1}{2}\left(1-\gamma_{5}\right) \tau_{1} \psi, \\
\left(Y_{R}\right)_{\mu} & =\bar{\psi} \gamma_{\mu} \frac{1}{2}\left(1+\gamma_{5}\right) \tau_{2} \psi, & -\left(Y_{L}\right)_{\mu} & =\bar{\psi} \gamma_{\mu} \frac{1}{2}\left(1-\gamma_{5}\right) \tau_{2} \psi, \\
\left(W_{R}\right)_{\mu} & =\bar{\psi} \gamma_{\mu} \frac{1}{2}\left(1+\gamma_{5}\right) \tau_{3} \psi, & \left(W_{L}\right)_{\mu} & =\bar{\psi} \gamma_{\mu} \frac{1}{2}\left(1-\gamma_{5}\right) \tau_{3} \psi .
\end{aligned}
$$

A unitary isospin transformation $\left(\psi_{p}, \psi_{n}\right) \rightarrow\left(\psi_{p}^{\prime}, \psi_{n}^{\prime}\right)$ leaves the time-like vector $\left(V_{R}\right)_{\mu}$ invariant, and transforms the vectors $\left(X_{R}\right)_{\mu},\left(Y_{R}\right)_{\mu},\left(W_{R}\right)_{\mu}$ into three orthogonal vectors within the spacelike subspace orthogonal to $\left(V_{R}\right)_{\mu}$, in a way which corresponds to a 3-dimensional rotation. The important point is that $\left(X_{R}\right)_{\mu},\left(Y_{R}\right)_{\mu},\left(W_{R}\right)_{\mu}$ are orthogonal vectors of equal length in space-time, and their transformation relates in this way isospin transformations directly with actual space-time rotations. Similar statements hold for the transformation of the vectors $\left(V_{L}\right)_{\mu},\left(X_{L}\right)_{\mu}$, $\left(Y_{L}\right)_{\mu},\left(W_{L}\right)_{\mu}$. A charge conjugation $\eta \rightleftarrows \xi$ interchanges the two frames $V_{R}, X_{R}, Y_{R}, W_{R}$ and $V_{L}, X_{L}, Y_{L}, W_{L}$. 


\section{Angles and Hyperbolic Angles Determined by the Invariants}

The relative orientations of the tetrads at a given point are determined by the invariants. They are closely connected with the way in which the tetrads are built up from 2-component spinors, and the simplest way to establish the linear relationships between the tetrad 4-vectors is with the help of the spinorial construction. The relationships will be established under the assumption that the relevant invariants are different from zero.

Three pairwise linearly independent spinors $\eta_{p}, \xi_{p}, \xi_{n}$ determine three tetrads $\left\{V_{p}, W_{p}, X_{p}, Y_{p}\right\},\left\{V_{+}, W_{+}, X_{+}, Y_{+}\right\}$and $\left\{V_{R}, W_{R}, \mathrm{X}_{R}, \mathrm{Y}_{R}\right\}$. The relationships between these will be studied in some detail. There are four different ways to select three 2-component spinors from $\eta_{p}, \eta_{n}, \xi_{p}, \xi_{n}$ and to form a 'triplet' of tetrads. The relationships between the tetrads of any other triplet will follow from the first investigated case by simple substitutions.

One can choose only two linearly independent 2-component spinors at a given point, and for instance $\eta_{p}, \eta_{n}$ can be expressed as linear combinations

$$
\begin{aligned}
& \eta_{p}=\alpha_{p p} \xi_{p}+\alpha_{p n} \xi_{n}, \\
& \eta_{n}=\alpha_{n p} \xi_{p}+\alpha_{n n} \xi_{n},
\end{aligned}
$$

of $\xi_{p}, \xi_{n}$. The complex coefficients $\alpha$ can be determined by taking the exterior product of these two equations with $\xi_{n}$ or $\xi_{p}$, and comparing the result with the expressions $(16 \mathrm{a}-\mathrm{c})$ of $\Omega$. One obtains

$$
\begin{array}{ll}
\alpha_{p p}=\frac{\Omega_{+}}{\Omega_{n}}, & \alpha_{p n}=-\frac{\Omega_{p}}{\Omega_{n}}, \\
\alpha_{n p}=\frac{\Omega_{n}}{\Omega_{R}}, & \alpha_{n n}=-\frac{\Omega_{-}}{\Omega_{R}} .
\end{array}
$$

With $\frac{1}{2} \Omega_{L}=\eta_{n} \times \eta_{p}$, the exterior product of the two equations $(20 \mathrm{a}, \mathrm{b})$ gives

$$
\Omega_{R} \Omega_{L}=\Omega_{+} \Omega_{-}-\Omega_{p} \Omega_{n}
$$

This relationship reduces the number of complex invariants $\Omega$ which can be chosen independently, from 6 to 5 .

If one defines hyperbolic angles $\chi$ by means of

so that

$$
|\Omega|=\exp (\chi),
$$

$$
\Omega=\exp (\chi) \exp (i \theta),
$$

the ratios $(20 c)$ depend only on the differences

$$
\begin{aligned}
& \mu_{p}=\chi_{p}-\chi_{R}, \quad \mu_{n}=\chi_{n}-\chi_{R}, \quad \mu_{+}=\chi_{+}-\chi_{R}, \quad \mu_{-}=\chi_{-}-\chi_{R}, \\
& \vartheta_{p}=\theta_{p}-\theta_{R}, \quad \vartheta_{n}=\theta_{n}-\theta_{R}, \quad \vartheta_{+}=\theta_{+}-\theta_{R}, \quad \vartheta_{-}=\theta_{-}-\theta_{R},
\end{aligned}
$$


of the hyperbolic angles and angles, and one can write

$$
\begin{array}{ll}
\frac{\Omega_{+}}{\Omega_{R}}=\exp \left(\mu_{+}\right) \exp \left(i \vartheta_{+}\right), & \frac{\Omega_{p}}{\Omega_{R}}=\exp \left(\mu_{p}\right) \exp \left(i \vartheta_{p}\right), \\
\frac{\Omega_{n}}{\Omega_{R}}=\exp \left(\mu_{n}\right) \exp \left(i \vartheta_{n}\right), & \frac{\Omega_{-}}{\Omega_{R}}=\exp \left(\mu_{-}\right) \exp \left(i \vartheta_{-}\right) .
\end{array}
$$

In order to express the vectors $V_{p}, W_{p}, X_{p}, Y_{p}$ in terms of $V_{R}, W_{R}$, $X_{R}, Y_{R}$, one can use the equations

$$
\begin{aligned}
& \frac{1}{2}\left(V_{p}+W_{p}\right)=R_{p}=\xi_{p}^{*} \sigma \xi_{p} \\
& \frac{1}{2}\left(V_{p}-W_{p}\right)=L_{p}=\eta_{p}^{*} \sigma \eta_{p} \\
& \frac{1}{2}\left(X_{p}+i Y_{p}\right)=Z_{p}=\xi_{p}^{*} \sigma \eta_{p}
\end{aligned}
$$

and express $\eta_{p}$ by means of $(20 \mathrm{a}, \mathrm{c})$. This leads to identities of the form

$$
\begin{aligned}
\Omega_{R} Z_{p} & =\Omega_{+} R_{p}-\Omega_{p} R_{+}, \\
\Omega_{R}^{*} \Omega_{R} L_{p} & =\Omega_{+}^{*} \Omega_{+} R_{p}+\Omega_{p}^{*} \Omega_{p} R_{n}-\Omega_{+}^{*} \Omega_{p} R_{+}-\Omega_{p}^{*} \Omega_{+} R_{-} .
\end{aligned}
$$

The relative orientation of the two tetrads can be expressed more immediately in terms of the unit vectors $v_{p}, w_{p}, x_{p}, y_{p}$ and $v_{R}, w_{R}, x_{R}, y_{R}$. With these, and the notation (23c), the equations $(24 a-c),(25 a, b)$ give

$$
\begin{aligned}
& \exp \left(\mu_{+}\right) \frac{1}{2}\left(v_{p}+w_{p}\right)=\exp \left(\mu_{+}-\mu_{p}\right) \frac{1}{2}\left(v_{R}+w_{R}\right), \\
& \exp \left(-\mu_{+}\right) \frac{1}{2}\left(v_{p}-w_{p}\right) \\
& =\left\{\exp \left(\mu_{+}-\mu_{p}\right) \frac{1}{2}\left(v_{R}+w_{R}\right)+\exp \left(\mu_{p}-\mu_{+}\right) \frac{1}{2}\left(v_{R}-w_{R}\right)\right\} \\
& \quad-\left\{\exp \left[-i\left(\vartheta_{+}-\vartheta_{p}\right)\right] \frac{1}{2}\left(x_{R}+i y_{R}\right)+\exp \left[i\left(\vartheta_{+}-\vartheta_{p}\right)\right] \frac{1}{2}\left(x_{R}-i y_{R}\right)\right\}, \\
& \exp \left(-i \vartheta_{+}\right) \frac{1}{2}\left(x_{p}+i y_{p}\right)=\exp \left(\mu_{+}-\mu_{p}\right) \frac{1}{2}\left(v_{R}+w_{R}\right) \\
& \quad-\exp \left[-i\left(\vartheta_{+}-\vartheta_{p}\right)\right] \frac{1}{2}\left(x_{R}+i y_{R}\right) .
\end{aligned}
$$

In introducing unit vectors $v(\mu), w(\mu)$ related to $v, w$ by means of a Lorentz transformation

$$
\begin{aligned}
v(\mu) & =v \cosh \mu+w \sinh \mu, & v(0) & =v, \\
w(\mu) & =v \sinh \mu+w \cosh \mu, & w(0) & =w
\end{aligned}
$$

in the $(v, w)$ plane, and similarly unit vectors $x(\vartheta), y(\vartheta)$ related to $x, y$ through a rotation

$$
\begin{array}{ll}
x(\vartheta)=x \cos \vartheta+y \sin \vartheta, & x(0)=x, \\
y(\vartheta)=-x \sin \vartheta+y \cos \vartheta, & y(0)=y
\end{array}
$$


in the dual $(x, y)$ plane, the corresponding real and complex null-vectors transform according to the equations

$$
\begin{aligned}
& \frac{1}{2}(v(\mu)+w(\mu))=\exp (\mu) \frac{1}{2}(v+w), \\
& \frac{1}{2}(v(\mu)-w(\mu))=\exp (-\mu) \frac{1}{2}(v-w), \\
& \frac{1}{2}(x(\vartheta) \pm i y(\vartheta))=\exp (\mp i \vartheta) \frac{1}{2}(x \pm i y) .
\end{aligned}
$$

In terms of such rotated unit vectors, the equations $(26 a, b, c)$ can be written in the form

$$
\begin{aligned}
& \frac{1}{2}\left\{v_{p}\left(\mu_{+}\right)+w_{p}\left(\mu_{+}\right)\right\}=\frac{1}{2}\left\{v_{R}\left(\mu_{+}-\mu_{p}\right)+w_{R}\left(\mu_{+}-\mu_{p}\right)\right\}, \\
& \frac{1}{2}\left\{v_{p}\left(\mu_{+}\right)-w_{p}\left(\mu_{+}\right)\right\}=v_{R}\left(\mu_{+}-\mu_{p}\right)-x_{R}\left(\vartheta_{+}-\vartheta_{p}\right), \\
& x_{p}\left(\vartheta_{+}\right)=\left\{v_{R}\left(\mu_{+}-\mu_{p}\right)+w_{R}\left(\mu_{+}-\mu_{p}\right)\right\}-x_{R}\left(\vartheta_{+}-\vartheta_{p}\right), \\
& y_{p}\left(\vartheta_{+}\right)=-y_{R}\left(\vartheta_{+}-\vartheta_{p}\right) .
\end{aligned}
$$

These equations give $v_{p}, w_{p}, x_{p}, y_{p}$ in function of $v_{R}, w_{R}, x_{R}, y_{R}$ with the intermediary of the equations $(27 \mathrm{c}, \mathrm{d}, \mathrm{e})$. Very little algebra is needed to invert the equations and express $v_{R}, w_{R}, x_{R}, y_{R}$ in terms of $v_{p}, w_{p}, x_{p}, y_{p}$. The angles $(23 \mathrm{a}, \mathrm{b})$ can be seen to appear directly as angles and hyperbolic angles of rotations and Lorentz transformations in the $(x, y)$ and $(v, w)$ planes.

In order to express the vectors $V_{+}, W_{+}, X_{+}, Y_{+}$in terms of $V_{R}, W_{R}$, $X_{R}, Y_{R}$, one can start from the equations

$$
\begin{aligned}
& \frac{1}{2}\left(V_{+}+W_{+}\right)=R_{n}=\xi_{n}^{*} \sigma \xi_{n}, \\
& \frac{1}{2}\left(V_{+}-W_{+}\right)=L_{p}=\eta_{p}^{*} \sigma \eta_{p}, \\
& \frac{1}{2}\left(X_{+}+i Y_{+}\right)=Z_{+}=\xi_{n}^{*} \sigma \eta_{p} .
\end{aligned}
$$

Proceeding as before, and using (20a, c), equation (25a) is replaced by

$$
\Omega_{R} Z_{+}=\Omega_{+} R_{-}-\Omega_{p} R_{n}
$$

which can be used together with $(25 \mathrm{~b})$. Introducing unit vectors and the notation $(23 \mathrm{c})$, the three equations $(29 \mathrm{a}, \mathrm{b}, \mathrm{c})$ lead to

$$
\begin{aligned}
& \frac{1}{2}\left\{v_{+}\left(\mu_{p}\right)+w_{+}\left(\mu_{p}\right)\right\}=\frac{1}{2}\left\{v_{R}\left(\mu_{+}-\mu_{p}\right)-w_{R}\left(\mu_{+}-\mu_{p}\right)\right\}, \\
& \frac{1}{2}\left\{v_{+}\left(\mu_{p}\right)-w_{+}\left(\mu_{p}\right)\right\}=v_{R}\left(\mu_{+}-\mu_{p}\right)-x_{R}\left(\vartheta_{+}-\vartheta_{p}\right), \\
& x_{+}\left(\vartheta_{p}\right)=-\left\{v_{R}\left(\mu_{+}-\mu_{p}\right)-w_{R}\left(\mu_{+}-\mu_{p}\right)\right\}+x_{R}\left(\vartheta_{+}-\vartheta_{p}\right), \\
& y_{+}\left(\vartheta_{p}\right)=-y_{R}\left(\vartheta_{+}-\vartheta_{p}\right) .
\end{aligned}
$$


The relationship between the tetrads $\left\{v_{p}, w_{p}, x_{p}, y_{p}\right\}$ and $\left\{v_{+}, w_{+}, x_{+}, y_{+}\right\}$ results from $(29 a-d)$ and $(30 a-d)$ in the form

$$
\begin{aligned}
& \frac{1}{2}\left\{v_{p}\left(\mu_{+}\right)-w_{p}\left(\mu_{+}\right)\right\}=\frac{1}{2}\left\{v_{+}\left(\mu_{p}\right)-w_{+}\left(\mu_{p}\right)\right\}, \\
& \frac{1}{2}\left\{v_{p}\left(\mu_{+}\right)+w_{p}\left(\mu_{+}\right)\right\}=v_{+}\left(\mu_{p}\right)+x_{+}\left(\vartheta_{p}\right), \\
& x_{p}\left(\vartheta_{+}\right)=\left\{v_{+}\left(\mu_{p}\right)-w_{+}\left(\mu_{p}\right)\right\}+x_{+}\left(\vartheta_{p}\right), \\
& y_{p}\left(\vartheta_{+}\right)=y_{+}\left(\vartheta_{p}\right) .
\end{aligned}
$$

\section{Symmetry of the Main Tetrad Planes}

With the abbreviated notation

$$
\begin{gathered}
v_{p}\left(\mu_{+}\right), w_{p}\left(\mu_{+}\right), x_{p}\left(\vartheta_{+}\right), y_{p}\left(\vartheta_{+}\right) \rightarrow v_{p}^{\prime}, w_{p}^{\prime}, x_{p}^{\prime}, y_{p}^{\prime} \\
v_{+}\left(\mu_{p}\right), w_{+}\left(\mu_{p}\right), x_{+}\left(\vartheta_{p}\right), y_{+}\left(\vartheta_{p}\right) \rightarrow v_{+}^{\prime}, w_{+}^{\prime}, x_{+}^{\prime}, y_{+}^{\prime} \\
v_{R}\left(\mu_{+}-\mu_{p}\right), w_{R}\left(\mu_{+}-\mu_{p}\right), x_{R}\left(\vartheta_{+}-\vartheta_{p}\right), y_{R}\left(\vartheta_{+}-\vartheta_{p}\right) \rightarrow v_{R}^{\prime}, w_{R}^{\prime}, x_{R}^{\prime}, y_{R}^{\prime}
\end{gathered}
$$

the relationships $(28 \mathrm{a}-\mathrm{d}),(30 \mathrm{a}-\mathrm{d}),(31 \mathrm{a}-\mathrm{d})$ between the dashed tetrads can be seen to be independent of the arguments $\mu, \vartheta$ which describe Lorentz transformations and rotations in the $(v, w)$ and $(x, y)$ planes. The same relationships could be derived by taking the special symmetric case $\Omega_{+}=\Omega_{p}=\Omega_{R}$, that is $\mu_{+}=\mu_{p}=\mu_{+}-\mu_{p}=0, \vartheta_{+}=\vartheta_{p}$ $=\vartheta_{+}-\vartheta_{p}=0$, and substituting accordingly $\eta_{p}=\xi_{p}-\xi_{n}$ into the equations $(24 a, b, c)$ and $(29 a, b, c)$. As it will be seen, the dashed tetrads are determined apart from signs of the vectors, by the three main tetrad planes $(v, w)$.

The $\left(v^{\prime}, w^{\prime}\right)$ planes are the same as the $(v, w)$ planes and the lines of intersection of the planes $\left(v_{p}, w_{p}\right),\left(v_{+}, w_{+}\right),\left(v_{R}, w_{R}\right)$ are given by the three null-vectors

$$
\begin{aligned}
& \frac{1}{2}\left(v_{p}^{\prime}+w_{p}^{\prime}\right)=\frac{1}{2}\left(v_{R}^{\prime}+w_{R}^{\prime}\right)=v_{+}^{\prime}+x_{+}^{\prime}, \\
& \frac{1}{2}\left(v_{p}^{\prime}-w_{p}^{\prime}\right)=\frac{1}{2}\left(v_{+}^{\prime}-w_{+}^{\prime}\right)=v_{R}^{\prime}-x_{R}^{\prime}, \\
& \frac{1}{2}\left(v_{+}^{\prime}+w_{+}^{\prime}\right)=\frac{1}{2}\left(v_{R}^{\prime}-w_{R}^{\prime}\right)=v_{p}^{\prime}-x_{p}^{\prime} .
\end{aligned}
$$

The three dual planes $\left(x_{p}, y_{p}\right),\left(x_{+}, y_{+}\right),\left(x_{R}, y_{R}\right)$, which are the same as the corresponding $\left(x^{\prime}, y^{\prime}\right)$ planes, have a common line of intersection defined by

$$
y_{p}^{\prime}=y_{+}^{\prime}=-y_{R}^{\prime} .
$$

The relationships $(28 a-c),(30 a-c),(31 a-c)$ between the vectors of the dashed tetrads involve only a 3 -dimensional subspace of space-time, which is orthogonal to this direction (33d) and has two space-like and one time-like dimensions. 
From the equations $(33 \mathrm{a}-\mathrm{c})$, the three space-like vectors $w_{p}^{\prime}, w_{+}^{\prime}, w_{R}^{\prime}$ can be expressed by the relationships

$$
\begin{aligned}
& w_{p}^{\prime}=\left(\frac{1}{2} v_{+}^{\prime}+x_{+}^{\prime}\right)+\frac{1}{2} w_{+}^{\prime}=\left(-\frac{1}{2} v_{R}^{\prime}+x_{R}^{\prime}\right)+\frac{1}{2} w_{R}^{\prime}, \\
& w_{+}^{\prime}=\left(\frac{1}{2} v_{p}^{\prime}-x_{p}^{\prime}\right)+\frac{1}{2} w_{p}^{\prime}=\left(-\frac{1}{2} v_{R}^{\prime}+x_{R}^{\prime}\right)-\frac{1}{2} w_{R}^{\prime}, \\
& w_{R}^{\prime}=-\left(\frac{1}{2} v_{p}^{\prime}-x_{p}^{\prime}\right)+\frac{1}{2} w_{p}^{\prime}=\left(\frac{1}{2} v_{+}^{\prime}+x_{+}^{\prime}\right)-\frac{1}{2} w_{+}^{\prime} .
\end{aligned}
$$

These show that the three vectors are in the same plane,

$$
w_{p}^{\prime}=w_{+}^{\prime}+w_{R}^{\prime}
$$

and, since they are unit vectors, they form with each other an angle of $60^{\circ}$ or $120^{\circ}$.

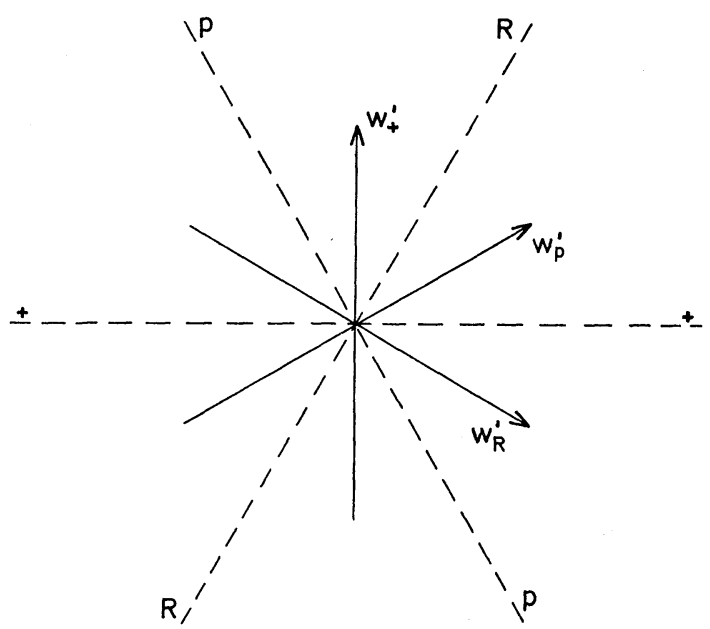

Fig. 1. Hexagonal symmetry of the lines of $w_{+}^{\prime}, w_{p}^{\prime}$ and $w_{R}^{\prime}$ which are in a common plane. The lines indicated by $++, p p, R R$ are the lines of intersection of the planes $\left(v_{+}, x_{+}\right),\left(v_{p}, x_{p}\right),\left(v_{R}, x_{R}\right)$ with the plane of the vectors $w^{\prime}$. The three planes intersect in a common line, are orthogonal to the plane of the vectors $w^{\prime}$, and halve the angle between two neighbouring lines $w^{\prime}$

The vector $w_{p}^{\prime}$ is orthogonal to the plane $\left(v_{p}^{\prime}, x_{p}^{\prime}\right)$ and to its line of intersection with this common $\left(w_{p}^{\prime}, w_{+}^{\prime}\right)$ plane of the three vectors. According to $(34 \mathrm{~b})$, this line of intersection is given by $\frac{1}{2} v_{p}^{\prime}-x_{p}^{\prime}$. With similar statements concerning $w_{+}^{\prime}$ and $w_{R}^{\prime}$, one can see that the three planes $\left(v_{p}^{\prime}, x_{p}^{\prime}\right),\left(v_{+}^{\prime}, x_{+}^{\prime}\right),\left(v_{R}^{\prime}, x_{R}^{\prime}\right)$ intersect the plane $\left(w_{p}^{\prime}, w_{+}^{\prime}\right)$ in lines which halve the angles between the lines given by $w_{p}^{\prime}, w_{+}^{\prime}, w_{R}^{\prime}$. (See Fig. 1.) The three planes $\left(v_{p}^{\prime}, x_{p}^{\prime}\right),\left(v_{+}^{\prime}, x_{+}^{\prime}\right),\left(v_{R}^{\prime}, x_{R}^{\prime}\right)$ intersect each other 
in a common line given by

$$
v_{p}^{\prime}-\frac{1}{2} x_{p}^{\prime}=v_{+}^{\prime}+\frac{1}{2} x_{+}^{\prime}=v_{R}^{\prime}-\frac{1}{2} x_{R}^{\prime}
$$

which is orthogonal to the plane $\left(w_{p}^{\prime}, w_{+}^{\prime}\right)$.

Each of the three planes contains one of the null vectors $(33 a, b, c)$. After normalisation, the orthogonal vectors $v_{p}^{\prime}-\frac{1}{2} x_{p}^{\prime}$ and $-\left(\frac{1}{2} v_{p}^{\prime}-x_{p}^{\prime}\right)$, are related to the unit vectors $v_{p}^{\prime}, x_{p}^{\prime}$ by a Lorentz transformation, and similar statements hold in the other two planes. One could bring the three dashed tetrads into coincidence by transforming $v_{p}^{\prime}, v_{+}^{\prime}, v_{R}^{\prime}$ into the direction (35) through such Lorentz transformations, with hyperbolic angles $\mu$ given by $\exp ( \pm \mu)=\sqrt{3}$, and then performing further rotations of $60^{\circ}$ in the orthogonal $\left(w_{p}^{\prime}, w_{+}^{\prime}\right)$ plane.

The plane $\left(v_{p}^{\prime}, w_{p}^{\prime}\right)$ intersects $\left(v_{+}^{\prime}, x_{+}^{\prime}\right)$ in the null-line (33a), and $\left(v_{R}^{\prime}, x_{R}^{\prime}\right)$ in the null-line $(33 \mathrm{~b})$. Its line of intersection with $\left(v_{p}^{\prime}, x_{p}^{\prime}\right)$ is of course $v_{p}^{\prime}$, and with $\left(w_{p}^{\prime}, w_{+}^{\prime}\right)$ it is $w_{p}^{\prime}$. Similarly, the line of intersection of $\left(v_{+}^{\prime}, w_{+}^{\prime}\right)$ with $\left(v_{p}^{\prime}, x_{p}^{\prime}\right)$ and with $\left(v_{R}^{\prime}, x_{R}^{\prime}\right)$ is one of the three null-lines, and so is that of $\left(v_{R}^{\prime}, w_{R}^{\prime}\right)$ with $\left(v_{p}^{\prime}, x_{p}^{\prime}\right)$ and with $\left(v_{+}^{\prime}, w_{+}^{\prime}\right)$. The plane connecting $w_{p}^{\prime}$ with the third of the null-lines, $(34 \mathrm{c})$, contains, on the other hand, both $x_{+}^{\prime}$ and $x_{R}^{\prime}$. The plane $\left(x_{+}^{\prime}, x_{R}^{\prime}\right)$ contains according to $(30 \mathrm{c})$ the nullline $(33 \mathrm{c})$, and the linear combination $w_{p}^{\prime}=\frac{1}{2} x_{+}^{\prime}+\frac{1}{2}\left(v_{+}^{\prime}+w_{+}^{\prime}\right)$ is in the same plane. Similarly, $w_{+}^{\prime}, x_{p}^{\prime}, x_{R}^{\prime}$ and the null-line (33a) are in one plane, and also $w_{R}^{\prime}, x_{+}^{\prime}, x_{p}^{\prime}$ and $(33 \mathrm{~b})$.

That the main tetrad planes $\left(v_{p}, w_{p}\right),\left(v_{+}, w_{+}\right),\left(v_{R}, w_{R}\right)$ determine the dashed tetrads apart from signs, can now be seen. The common line of intersection of the dual planes $\left(x_{p}, y_{p}\right),\left(x_{+}, y_{+}\right),\left(x_{R}, y_{R}\right)$ gives the line of $y_{p}^{\prime}=y_{+}^{\prime}=-y_{R}^{\prime}$, and the orthogonal directions in these planes give $x_{p}^{\prime}, x_{+}^{\prime}, x_{R}^{\prime}$. The lines of intersection of $\left(x_{+}^{\prime}, x_{R}^{\prime}\right)$ with $\left(v_{p}, w_{p}\right)$, of $\left(x_{p}^{\prime}, x_{R}^{\prime}\right)$ with $\left(v_{+}, w_{+}\right)$and of $\left(x_{p}^{\prime}, x_{+}^{\prime}\right)$ with $\left(v_{R}, w_{R}\right)$ give $w_{p}^{\prime}, w_{+}^{\prime}, w_{R}^{\prime}$. The planes $\left(x_{p}^{\prime}, x_{+}^{\prime}\right)$ and $\left(x_{p}^{\prime}, x_{R}^{\prime}\right)$ contain each one of the three null-vectors. Connecting $x_{p}^{\prime}$ with the third null-vector gives the plane which cuts $\left(v_{p}, w_{p}\right)$ at the line of $v_{p}^{\prime}$. The analogous plane through $x_{+}^{\prime}$ gives $v_{+}^{\prime}$, and through $x_{R}^{\prime}, v_{R}^{\prime}$.

Three pairwise linearly independent 2-component spinors determine three real null-vectors and the related null-lines. The inverse construction of three spinors from three null-lines is by no means unique, but there are always many ways to find three pairwise linearly independent spinors which give the three null-lines and for which the previous constructions follow. By explicit construction, and so not in the simplest possible way, some simple geometrical theorems in space-time are therefore also established. These can be summarised as follows. Given any three null-lines through a point $O$ in space-time, they determine pairwise three 'main' planes. The three dual planes to these at $O$ have a common 
space-like line of intersection, $y$. There exists a time-like direction $t$ at $O$, orthogonal to $y$, and a space-like plane $S$ going through $O$, orthogonal both to $t$ and to $y$, in such a way that the lines of intersection $w$ of $S$ with the three main planes are in the symmetric hexagonal position, intersecting under an angle $2 \pi / 3$ or $2 \pi / 6$. The three planes through $t$ and one of the three null-lines halve the angle between two neighbouring lines $w$, and are perpendicular to the third $w$, to $S$, and to $y$. (See Fig. 1.)

\section{Substitutions and Charge Conjugation}

The relative position of the three tetrads $\left\{V_{-}, W_{-}, X_{-}, Y_{-}\right\}$, $\left\{V_{n}, W_{n}, X_{n}, Y_{n}\right\}$ and $\left\{V_{R}, W_{R}, Y_{R}, Y_{R}\right\}$ which are given by $\eta_{n}, \xi_{p}, \xi_{n}$, follows from the previous considerations in replacing $\eta_{p}$ by $\eta_{n}$. From the definitions, an interchange of $\eta_{p}$ and $\eta_{n}$ means

$$
\begin{aligned}
V_{p}, W_{p}, X_{p}, Y_{p} \rightleftarrows V_{-}, W_{-}, X_{-}, Y_{-}, & & \Omega_{p} \rightleftarrows \Omega_{-}, \\
V_{+}, W_{+}, X_{+}, Y_{+} \rightleftarrows V_{n}, W_{n}, X_{n}, Y_{n}, & & \Omega_{+} \rightleftarrows \Omega_{n}, \\
V_{R}, W_{R}, X_{R}, Y_{R} \rightleftarrows V_{R}, W_{R}, X_{R}, Y_{R}, & & \Omega_{R} \rightleftarrows \Omega_{R} .
\end{aligned}
$$

The relationships $(28 a-d),(30 a-d),(31 a-d)$ give, therefore, similar relationships for the new triplet of tetrads by a simple interchange of symbols:

$$
\begin{gathered}
v_{p}\left(\mu_{+}\right), w_{p}\left(\mu_{+}\right), x_{p}\left(\vartheta_{+}\right), y_{p}\left(\vartheta_{+}\right) \rightleftarrows v_{-}\left(\mu_{n}\right), w_{-}\left(\mu_{n}\right), x_{-}\left(\vartheta_{n}\right), y_{-}\left(\vartheta_{n}\right) \\
v_{+}\left(\mu_{p}\right), w_{+}\left(\mu_{p}\right), x_{+}\left(\vartheta_{p}\right), y_{+}\left(\vartheta_{p}\right) \rightleftarrows v_{n}\left(\mu_{-}\right), w_{n}\left(\mu_{-}\right), x_{n}\left(\vartheta_{-}\right), y_{n}\left(\vartheta_{-}\right), \\
v_{R}\left(\mu_{+}-\mu_{p}\right), w_{R}\left(\mu_{+}-\mu_{p}\right), x_{R}\left(\vartheta_{+}-\vartheta_{p}\right), y_{R}\left(\vartheta_{+}-\vartheta_{p}\right) \\
\rightleftarrows v_{R}\left(\mu_{n}-\mu_{-}\right), w_{R}\left(\mu_{n}-\mu_{-}\right), x_{R}\left(\vartheta_{n}-\vartheta_{-}\right), y_{R}\left(\vartheta_{n}-\vartheta_{-}\right) .
\end{gathered}
$$

The relationships between the elements of the other two triplets of tetrads, determined by $\xi_{p}, \eta_{p}, \eta_{n}$ and by $\xi_{n}, \eta_{p}, \eta_{n}$, can be obtained by means of charge conjugation, $\xi_{p} \rightleftarrows \eta_{p}, \xi_{n} \rightleftarrows \eta_{n}$. With this interchange one has

and

$$
\begin{array}{lll}
R_{p} \rightleftarrows L_{p}, & R_{n} \rightleftarrows L_{n}, & R_{+} \rightleftarrows L_{-}, \\
Z_{p} \rightleftarrows Z_{p}^{*}, & Z_{n} \rightleftarrows Z_{n}^{*}, & Z_{+} \rightleftarrows Z_{-}^{*},
\end{array}
$$

$$
\begin{aligned}
& V_{p}, W_{p}, X_{p}, Y_{p} \rightleftarrows V_{p},-W_{p}, X_{p},-Y_{p}, \quad \Omega_{p} \rightleftarrows-\Omega_{p}, \\
& V_{n}, W_{n}, X_{n}, Y_{n} \rightleftarrows V_{n},-W_{n}, X_{n},-Y_{n}, \quad \Omega_{n} \rightleftarrows-\Omega_{n}, \\
& V_{+}, W_{+}, X_{+}, Y_{+} \rightleftarrows V_{-},-W_{-}, X_{-},-Y_{-}, \quad \Omega_{+} \rightleftarrows-\Omega_{-} \text {, } \\
& V_{R}, W_{R}, X_{R}, Y_{R} \rightleftarrows V_{L}, W_{L}, X_{L}, Y_{L}, \quad \Omega_{R} \rightleftarrows-\Omega_{L} \text {. }
\end{aligned}
$$

The definition of $\Omega_{L}$ was chosen in such a way that $\Omega_{R} \rightleftarrows-\Omega_{L}$, and accordingly $\Omega_{p} / \Omega_{R} \rightleftarrows \Omega_{p} / \Omega_{L}, \Omega_{n} / \Omega_{R} \rightleftarrows \Omega_{n} / \Omega_{L}, \Omega_{ \pm} / \Omega_{R} \rightleftarrows \Omega_{\mp} / \Omega_{L}$. For the 
angles and hyperbolic angles defined by $\Omega=\exp (\chi) \exp (i \theta)$, this means $\chi_{p}-\chi_{R} \rightleftarrows \chi_{p}-\chi_{L}, \quad \chi_{n}-\chi_{R} \rightleftarrows \chi_{n}-\chi_{L}, \quad \chi_{ \pm}-\chi_{R} \rightleftarrows \chi_{\mp}-\chi_{L}$, $\theta_{p}-\theta_{R} \rightleftarrows \theta_{p}-\theta_{L}, \quad \theta_{n}-\theta_{R} \rightleftarrows \theta_{n}-\theta_{L}, \quad \theta_{ \pm}-\theta_{R} \rightleftarrows \theta_{\mp}-\theta_{L}$.

The Lorentz transformations and rotations $(27 \mathrm{a}, \mathrm{b})$ in the $(v, w)$ and $(x, y)$ planes can be seen to be invariant with respect to a simultaneous change of the sign of $w$ and $\mu$ or of $y$ and $\vartheta$. If one defines rotated unit vectors with the angles

$$
\begin{array}{ll}
\bar{\mu}_{p}=-\left(\chi_{p}-\chi_{L}\right), & \bar{\mu}_{n}=-\left(\chi_{n}-\chi_{L}\right), \\
\bar{\mu}_{+}=-\left(\chi_{+}-\chi_{L}\right), & \bar{\mu}_{-}=-\left(\chi_{-}-\chi_{L}\right), \\
\bar{\vartheta}_{p}=-\left(\theta_{p}-\theta_{L}\right), & \bar{\vartheta}_{n}=-\left(\theta_{n}-\theta_{L}\right), \\
\bar{\vartheta}_{+}=-\left(\theta_{+}-\theta_{L}\right), & \bar{\vartheta}_{-}=-\left(\theta_{-}-\theta_{L}\right)
\end{array}
$$

the relative minus sign in comparison with the definitions $(23 \mathrm{a}, \mathrm{b})$ compensates for the changes in sign of $W$ and $Y$ in the transitions $(38 \mathrm{c}, \mathrm{d}, \mathrm{e})$. The connection between the three tetrads $\left\{v_{p}, w_{p}, x_{p}, y_{p}\right\}$, $\left\{v_{-}, w_{-}, x_{-}, y_{-}\right\}$and $\left\{v_{L}, w_{L}, x_{L}, y_{L}\right\}$ is obtained therefore from the relationships $(28 \mathrm{a}-\mathrm{d}),(30 \mathrm{a}-\mathrm{d}),(31 \mathrm{a}-\mathrm{d})$ by the interchange

$$
\begin{gathered}
v_{p}\left(\mu_{+}\right), w_{p}\left(\mu_{+}\right), x_{p}\left(\vartheta_{+}\right), y_{p}\left(\vartheta_{+}\right) \rightleftarrows v_{p}\left(\bar{\mu}_{-}\right), w_{p}\left(\bar{\mu}_{-}\right), x_{p}\left(\bar{\vartheta}_{-}\right), y_{p}\left(\bar{\vartheta}_{-}\right), \\
v_{+}\left(\mu_{p}\right), w_{+}\left(\mu_{p}\right), x_{+}\left(\vartheta_{p}\right), y_{+}\left(\vartheta_{p}\right) \rightleftarrows v_{-}\left(\bar{\mu}_{p}\right), w_{-}\left(\bar{\mu}_{p}\right), x_{-}\left(\bar{\vartheta}_{p}\right), y_{-}\left(\bar{\vartheta}_{p}\right), \\
v_{R}\left(\mu_{+}-\mu_{p}\right), w_{R}\left(\mu_{+}-\mu_{p}\right), x_{R}\left(\vartheta_{+}-\vartheta_{p}\right), y_{R}\left(\vartheta_{+}-\vartheta_{p}\right) \\
\rightleftarrows v_{L}\left(\bar{\mu}_{p}-\bar{\mu}_{-}\right), w_{L}\left(\bar{\mu}_{p}-\bar{\mu}_{-}\right), x_{L}\left(\bar{\vartheta}_{p}-\bar{\vartheta}_{-}\right), y_{L}\left(\bar{\vartheta}_{p}-\bar{\vartheta}_{-}\right) .
\end{gathered}
$$

There is a different sign in the argument of the vectors of the two sides of $(41 \mathrm{c})$, in accordance with the definition of the angles and with the transformation (38f).

Similarly, the equations connecting the tetrads $\left\{v_{+}, w_{+}, x_{+}, y_{+}\right\}$, $\left\{v_{n}, w_{n}, x_{n}, y_{n}\right\}$ and $\left\{v_{L}, w_{L}, x_{L}, y_{L}\right\}$ can be obtained from

$$
\begin{aligned}
& v_{-}\left(\mu_{n}\right), w_{-}\left(\mu_{n}\right), x_{-}\left(\vartheta_{n}\right), y_{-}\left(\vartheta_{n}\right) \rightleftarrows v_{+}\left(\bar{\mu}_{n}\right), w_{+}\left(\bar{\mu}_{n}\right), x_{+}\left(\bar{\vartheta}_{n}\right), y_{+}\left(\bar{\vartheta}_{n}\right) \\
& v_{n}\left(\mu_{-}\right), w_{n}\left(\mu_{-}\right), x_{n}\left(\vartheta_{-}\right), y_{n}\left(\vartheta_{-}\right) \rightleftarrows v_{n}\left(\bar{\mu}_{+}\right), w_{n}\left(\bar{\mu}_{+}\right), x_{n}\left(\bar{\vartheta}_{+}\right), y_{n}\left(\bar{\vartheta}_{+}\right) \\
& v_{R}\left(\mu_{n}-\mu_{-}\right), w_{R}\left(\mu_{n}-\mu_{-}\right), x_{R}\left(\vartheta_{n}-\vartheta_{-}\right), y_{R}\left(\vartheta_{n}-\vartheta_{-}\right) \\
& \rightleftarrows v_{L}\left(\bar{\mu}_{+}-\bar{\mu}_{n}\right), w_{L}\left(\bar{\mu}_{+}-\bar{\mu}_{n}\right), x_{L}\left(\bar{\vartheta}_{+}-\bar{\vartheta}_{n}\right), y_{L}\left(\bar{\vartheta}_{+}-\bar{\vartheta}_{n}\right),
\end{aligned}
$$

combined with the substitutions $(37 \mathrm{a}, \mathrm{b}, \mathrm{c})$. The angles $\bar{\mu}, \bar{\vartheta}$ in the arguments of the rotated unit vectors are of course not independent from the angles $\mu, \vartheta$, since from the definitions $(23 a, b),(40 a, b)$, one has

$$
\begin{aligned}
& \mu_{p}+\bar{\mu}_{p}=\mu_{n}+\bar{\mu}_{n}=\mu_{+}+\bar{\mu}_{+}=\mu_{-}+\bar{\mu}_{-}=\chi_{L}-\chi_{R}, \\
& \vartheta_{p}+\bar{\vartheta}_{p}=\vartheta_{n}+\bar{\vartheta}_{n}=\vartheta_{+}+\bar{\vartheta}_{+}=\vartheta_{-}+\bar{\vartheta}_{-}=\theta_{L}-\theta_{R} .
\end{aligned}
$$


Both $\mu, \vartheta$ and $\bar{\mu}, \bar{\vartheta}$ are abbreviations for differences of angles, in a more symmetric way one could express the arguments of the vectors of each rotated tetrad within a triplet by means of the difference of the two angles $\theta$ or two hyperbolic angles $\chi$ defined by the two invariants $\Omega$ related to the other two tetrads.

The main planes $(v, w)$ of the 6 tetrads are determined by 4 real nulllines. The four different ways of selecting three of these gives the four tetrad triplets. In the general case, the $(x, y)$ planes determine a different common line of intersection for each triplet, and each triplet has its symmetry plane discussed in the previous section. If one wants to express directly the relationship between the vectors of two tetrads which are not members of a common triplet, for instance $\left\{v_{p}, w_{p}, x_{p}, y_{p}\right\}$ and $\left\{v_{n}, w_{n}, x_{n}, y_{n}\right\}$, or $\left\{v_{R}, w_{R}, x_{R}, y_{R}\right\}$ and $\left\{v_{L}, w_{L}, x_{L}, y_{L}\right\}$, this can be done in two different ways with the intermediary of a third tetrad which forms a triplet with both of them.

If one considered quarks, one would have to take a 12-component spinor

$$
\psi=\left(\psi_{p}, \psi_{n}, \psi_{\Lambda}\right)
$$

instead of an 8-component $\psi$. The related six 2-component spinors would define $\left(\begin{array}{l}6 \\ 2\end{array}\right)=15$ complex invariants $\Omega$ and 15 tetrads. The relationships between the quantities determined for instance by $\psi_{p}$ and $\psi_{\Lambda}$ would be formally the same as between those determined by $\psi_{p}, \psi_{n}$. The tetrad triplet determined by $\xi_{p}, \xi_{n}, \xi_{A}$ would have one tetrad, given by $\xi_{p}, \xi_{n}$, which relates isospin transformations to rotations of its space-like axes, one tetrad, given by $\xi_{p}, \xi_{A}$, which relates $U$-spin transformations with space-like rotations, and one, given by $\xi_{n}, \xi_{A}$ which does the same for $V$-spin transformations. Similar statements would hold for the tetrad triplet determined by $\eta_{p}, \eta_{n}, \eta_{\Lambda}$. Under unitary $S U_{3}$ transformations of $\xi_{p}, \xi_{n}, \xi_{\Lambda}$, the sum of the 3 real null-vectors $R_{p}, R_{n}, R_{A}$ would remain invariant, and the 3 real null-vectors and $2 \times 3$ complex null-vectors of the tetrad triplet would transform as an $\mathrm{SU}_{3}$ singlet and octet.

Through the spinorial construction and the transformation of its null-vectors, a tetrad triplet connects the basic $S U_{3}$ octet transformations to local space-time transformations. In the course of these a tetrad triplet transforms into a tetrad triplet through rotations and Lorentz transformations of the tetrads, together with a dilatation of the common length $|\Omega|^{2}$ of the four vectors of a tetrad, the sum of the three $|\Omega|^{2}$-s remaining invariant.

If one considered a 16-component spinor

$$
\psi=\left(\psi_{p}, \psi_{n}, \psi_{e}, \psi_{v}\right),
$$


the eight 2-component spinors would define $\left(\begin{array}{l}8 \\ 2\end{array}\right)=28$ complex invariants and 28 tetrads. These could be grouped into $\left(\begin{array}{l}8 \\ 3\end{array}\right)=56$ tetrad triplets. The relationships within each tetrad triplet would be exactly the same as discussed previously. The 4-vectors of any of the tetrads could be expressed in terms of the 4-vectors of any other tetrad either within a triplet, or with the intermediary of another tetrad which shares a triplet with both of them.

\section{Appendix. Relationships with 6-Vector Densities}

The 6-vector densities related to a 4-component spinor are given by the real and imaginary parts of the complex self-dual tensor

$$
\begin{aligned}
& M_{j k}=\bar{\psi} \gamma_{l} \gamma_{0} \frac{1}{2}\left(1+\gamma_{5}\right) \psi, \\
& M_{j k}^{*}=\bar{\psi} \gamma_{l} \gamma_{0} \frac{1}{2}\left(1-\gamma_{5}\right) \psi,
\end{aligned}
$$

where $j, k, l$ is one of the three cyclic permutations of $1,2,3$. In terms of the spinor components of $\psi=(\xi, \tilde{\eta})$ one has

$$
\begin{aligned}
& M_{j k}=-\tilde{\eta}^{*} \sigma_{l} \xi, \\
& M_{j k}^{*}=-\xi^{*} \sigma_{l} \tilde{\eta} .
\end{aligned}
$$

In terms of the arbitrary 2-component spinors $\alpha, \beta, \alpha^{\prime}, \beta^{\prime}$ which enter into the definition $(7 \mathrm{a}, \mathrm{b})$ of the null-vectors $U_{\mu}=U_{\mu}\left(\alpha^{*}, \beta\right)$, $U_{\mu}^{\prime}=U_{\mu}\left(\alpha^{*}, \beta^{\prime}\right)$, one can write

$$
\begin{aligned}
& M_{12}\left(\alpha, \alpha^{\prime}\right)=-\tilde{\alpha}^{*} \sigma_{3} \alpha^{\prime}=\alpha_{1} \alpha_{2}^{\prime}+\alpha_{2} \alpha_{1}^{\prime}, \\
& M_{23}\left(\alpha, \alpha^{\prime}\right)=-\tilde{\alpha}^{*} \sigma_{1} \alpha^{\prime}=-\alpha_{1} \alpha_{1}^{\prime}+\alpha_{2} \alpha_{2}^{\prime}, \\
& M_{31}\left(\alpha, \alpha^{\prime}\right)=-\tilde{\alpha}^{*} \sigma_{2} \alpha^{\prime}=\frac{1}{i}\left(\alpha_{1} \alpha_{1}^{\prime}+\alpha_{2} \alpha_{2}^{\prime}\right),
\end{aligned}
$$

and similar definitions for $M_{j k}\left(\beta, \beta^{\prime}\right)$. Whereas the invariants

$$
\frac{1}{2} \Omega\left(\alpha, \alpha^{\prime}\right)=\left|\begin{array}{ll}
\alpha_{1} & \alpha_{2} \\
\alpha_{1}^{\prime} & \alpha_{2}^{\prime}
\end{array}\right|
$$

are antisymmetric products of the two spinors $\alpha, \alpha^{\prime}$, the tensor $M_{j k}$ represents their symmetric product, $M_{j k}\left(\alpha, \alpha^{\prime}\right)=M_{j k}\left(\alpha^{\prime}, \alpha\right)$.

With very little algebra, one finds the identities

$$
\begin{aligned}
& \frac{1}{2 i}\left\{\Omega^{*}\left(\alpha, \alpha^{\prime}\right) M_{j k}\left(\beta, \beta^{\prime}\right)-\Omega\left(\beta, \beta^{\prime}\right) M_{j k}^{*}\left(\alpha, \alpha^{\prime}\right)\right\} \\
& =U_{j}\left(\alpha^{*}, \beta^{\prime}\right) U_{k}\left(\alpha^{*}, \beta\right)-U_{k}\left(\alpha^{*}, \beta^{\prime}\right) U_{j}\left(\alpha^{*}, \beta\right), \\
& \frac{1}{2}\left\{\Omega^{*}\left(\alpha, \alpha^{\prime}\right) M_{j k}\left(\beta, \beta^{\prime}\right)+\Omega\left(\beta, \beta^{\prime}\right) M_{j k}^{*}\left(\alpha, \alpha^{\prime}\right)\right\} \\
& =U_{0}\left(\alpha^{*}, \beta^{\prime}\right) U_{l}\left(\alpha^{*}, \beta\right)-U_{l}\left(\alpha^{*}, \beta^{\prime}\right) U_{0}\left(\alpha^{*}, \beta\right) .
\end{aligned}
$$


With the expressions (A. 2a-d), and similar ones for $U_{\mu}$, through cancellations each side of the two equations reduces to only two terms.

For the tensor components (A. $1 \mathrm{a}-\mathrm{d}$ ), one obtains with $\alpha=\beta=\xi$, $\alpha^{\prime}=\beta^{\prime}=\eta$,

$$
\begin{aligned}
\frac{1}{2 i}\left(\Omega^{*} M_{j k}-\Omega M_{j k}^{*}\right) & =L_{j} R_{k}-L_{k} R_{j}=\frac{1}{2}\left(V_{j} W_{k}-V_{k} W_{j}\right), \\
\frac{1}{2}\left(\Omega^{*} M_{j k}+\Omega M_{j k}^{*}\right) & =L_{0} R_{l}-L_{l} R_{0}=\frac{1}{2}\left(V_{0} W_{l}-V_{l} W_{0}\right) .
\end{aligned}
$$

For $\Omega \neq 0$, the equations give $M_{j k}$ in terms of $V_{\mu}, W_{\mu}$ and $\Omega$.

For an 8-component spinor, the identities (A. $3 a, b)$ give the tensorial densities in terms of the corresponding larger number of vectors and invariants.

\title{
References
}

1. Darwin, C. G.: Proc. Roy. Soc. A 120, 621 (1928).

2. Fock, V.: Z. f. Physik 57, 261 (1929).

3. Pauli, W.: Ann. Inst. Henri Poincaré 6, 109 (1936).

4. DE Broglit, L.: L'électron magnétique, Paris: Hermann 1934.

5. Proca, Al.: Ann. de Physique 20, 347 (1933).

6. Yvon, J.: C. R. 205, 1367 (1937); J. de Physique (8) 1, 18 (1940); Revue Scientifique 79, 209 (1941).

7. Petiad, G.: J. de Math. 25, 335 (1946); 26, 1 (1947).

8. Costa de Beadregard, O.: J. de Math. 22, 85 (1943).

9. Aymard, A.: C. R. 243, 885, 1100, 1198 (1956); 244, 3133 (1957).

10. Uhlenbeck, G. E., and O. Laporte: Phys. Rev. 37, 1380, 1552 (1931).

11. Whittaker, E. T.: Proc. Roy. Soc. A 158, 38 (1937).

12. Ruse, H. S.: Proc. Roy. Soc. Edinburgh 57, 97 (1937).

13. Kofink, W.: Ann. d. Physik [5] 30, 91 (1937); 38, 421, 436, 565, 583 (1940).

14. Takabayasi, T.: Suppl. Progr. Theor. Phys., No. 4 (1957); C. R. 246, 64 (1958); Nucl. Phys. 6, 477 (1958).

15. Frenz, M.: Z. f. Physik 104, 553 (1937).

16. CASE, K. M.: Phys. Rev. 97, 810 (1955).

17. Charap, J. M., and P. T. Matthews: Proc. Roy. Soc. A, 286, 300 (1965).

18. Stapp, H. P.: Phys. Rev. 125, 2160 (1962), Appendix J.

\author{
J. G. Valatin, \\ Department of Physics \\ Queen Mary College \\ University of London \\ Mile End Road \\ London E. 1., England
}

